\title{
What's new in musculoskeletal ultrasound in pediatric rheumatology?
}

\author{
Mihaela Spârchez ${ }^{1}$, Daniela Fodor ${ }^{2}$
}

${ }^{1} 2^{\text {nd }}$ Pediatric Department, ${ }^{2} 2^{\text {nd }}$ Internal Medicine Department, University of Medicine and Pharmacy, „Iuliu Hatieganu", Cluj-Napoca, România

\begin{abstract}
Musculoskeletal ultrasound (MSUS) has become almost indispensable in the rheumatology settings nowadays, allowing early diagnosis, careful guidance during procedures such as joint injections and therapy monitoring. Nonetheless, the applicability of MSUS in pediatric population is still limited. Recently, a standardized MSUS examination procedure in pediatric patients with rheumatic diseases, definitions for synovitis and the sonographic features of joints in healthy children has been developed. Also, important data on age-related vascularization and ossification of joints in children have been published. Much work still needs to be done in the field. As juvenile idiopathic arthritis seems to be the most common use of MSUS in pediatric rheumatology, specific definitions and assessment techniques for enthesitis, tenosynovitis, bone and cartilage damage in children are very much expected. In this article, we will review briefly the current evidence-based knowledge regarding MSUS potential applications in the pediatric rheumatology clinical practice, along with an overview of the recent information about US appearance of musculoskeletal structures in healthy children.
\end{abstract}

Keywords: musculoskeletal ultrasound; pediatric rheumatology; juvenile idiopathic arthritis

Musculoskeletal ultrasound (MSUS) is a useful tool with multiple applications in rheumatology and therefore rheumatologists have been using it for many years as an extension of the clinical examination. It has become almost indispensable in the rheumatology settings nowadays, allowing early diagnosis and careful guidance during procedures such as joint injections and therapy monitoring [1].

Nonetheless, the applicability of MSUS in the pediatric population is still limited. Recently, there has been an increasing interest among doctors (pediatricians, radiologists and pediatric rheumatologists) in using this imaging technique in the daily pediatric practice. Collado et al proposed recently a standardized MSUS examination procedure in the pediatric population with rheumatic disease [2]. Definitions for synovitis [3] and the

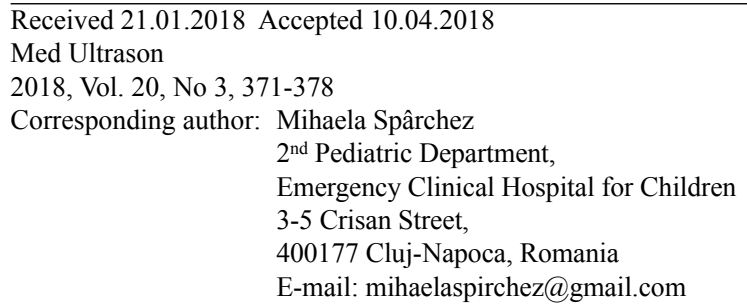

sonographic features of joints in healthy children have been developed [4]. These advances can bring about significant changes in practice by encouraging physicians to adhere to proper US techniques and it can facilitate better interpretation of their findings. Although important steps have been achieved recently, much work still needs to be done. This is undoubtedly important since the adult validated MSUS scoring systems cannot be used in children.

We will review briefly the current evidence-based knowledge regarding MSUS potential applications in the pediatric rheumatology clinical practice, along with an overview of recent information regarding US appearance of musculoskeletal structures in healthy children.

\section{MSUS advantages and disadvantages in children}

MSUS has important advantages that makes it applicable for the evaluation of pediatric musculoskeletal conditions. It is a noninvasive, safe, highly accessible and low-cost method, that can be easily and rapidly performed even in children. It can be repeated as many times as required, in combination with physical examinations, in an interactive way. In comparison with Magnetic Resonance Imaging (MRI), the duration of MSUS examina- 
tion is much shorter and US offers the option to evaluate more anatomical regions/joints during one session. Another advantage over MRI is that US examinations do not require sedation or general anesthesia. The US dynamic examinations are more captivating for children, especially for the very young ones that can easily lose patience [4-6].

The main disadvantage of MSUS is its operator dependence. It requires a good practical training and solid theoretical knowledge regarding age-related aspects of the growing skeleton. Another drawback includes various limits related to the impossibility to visualize intraosseous changes and the restricted acoustic windows of some regions $[5,6]$.

Acknowledging the advantages and benefits of MSUS in the clinical care of children with different MSK conditions, it is expected that more pediatric rheumatologists will actively use it in their daily practice. Consequently, for this reason, a pediatric sub-task force of the international Outcome Measures in Rheumatology Clinical Trials (OMERACT) US working group was created in order to validate the use of US in the assessment of joints in healthy children [7]. Moreover, "points to consider" were elaborated in 2015 by the European League Against Rheumatism (EULAR) and the Paediatric Rheumatology European Society (PreS) group of experts for the use of imaging, namely US and MRI, in the diagnosis and management of juvenile idiopathic arthritis (JIA) in clinical practice [8].

\section{The examination technique}

Over the past years, there has been significant technological improvements within the equipment allowing higher resolution images with better structural information. High multi-frequency (generally $\geq 12 \mathrm{MHz}$ ) linear transducers are needed for scanning musculoskeletal structures, adapted to age and examined area (higher frequencies for more superficial areas). For assessing small joints in children, it is preferably to use hockey stick transducers. The settings for Doppler ultrasound (power or color) need to be adjusted in order to detect low velocity flow. An appropriate choice of transducer's dimensions and frequency with a generous quantity of ultrasound gel has made stand-off pads unnecessary $[9,10]$.

Positioning the pediatric patients for MSUS exam depends on the area of study and the age of the patient. Older children may be examined in the same positions as adults, but for very young children the examination may be challenging. Assistance from the parent to hold and comfort the child is generally required or different tricks, in order to captivate the children's attention during the process and keep them relaxed (background music, friendly environment, toys, TV, screen savers etc), may be used. Small pillows or paper rolls may be placed under the joints in order to obtain the optimal position. Motion artefacts may result if the children move during the examination and that can be very misleading, especially for Doppler analysis. The scanning should involve bilateral regions, for a proper interpretation of the MSUS findings. Last but not least, in order to maintain calm, parents may need to be informed constantly regarding the whole procedure and the meanings of your findings. Reporting the results may be done after finishing the session [10].

\section{US appearance of musculoskeletal structures in healthy children}

Knowledge about normal US appearance of musculoskeletal system at different ages is very important before going into the pathological details. For this reason, the pediatric sub-task force of the OMERACT working group have made considerable efforts so far to define the US characteristics of joints in healthy children [4], to standardize the scanning procedure for the pediatric population [2] and to assess normal vascularization and ossification of joints in healthy children [7].

Bone formation is a process of endochondral ossification that begins during the fetal phase of life and continues gradually untill complete skeletal maturation at puberty. Osteogenesis initiates at the primary ossification site at the center of the diaphysis and later at the secondary (epiphyseal) ossification center. During this process, the cartilage, an avascular tissue, is replaced by bone, and a new structure, the growth plate (the epiphyseal plate, physis or metaphysis) is formed between the diaphysis and the epiphysis. The complete fusion of epiphysis and diaphysis is seen when the growth ends. The process of endochondral ossification is dependent upon neovascularization $[11,12]$. Normal maturation develops in a predefined sequence and skeletal maturation can be compared with normal age-related standards.

These characteristics of meta-epiphyseal regions of the bones in the growing child define the appearance of bony landmarks used for scanning synovial joints in children. Therefore, new definitions have had to be established, separate from the ones used for adults. After carrying out the consensus process and validation, a group of international experts published in 2015 definitions on the normal hyaline cartilage, the epiphyseal secondary ossification center, the normal joint capsule, the articular bone surface and the synovial membrane[4].

A preliminary semi-quantitative score has been developed recently [7] for the US ossification grading of 
the epiphyseal secondary ossification center. According to this score, ossification can be grade in four phases ( 0 to $3)$. Grade 0 has been used for the non-ossified epiphyseal bone, short bones or patella and grade 3 for the complete ossification. The size of the ossification center correlates with age and skeletal maturity $[13,14]$.

Thickness of joint cartilage is also related to age, sex, specific joint and not surprisingly, the occurrence of a pathologic process. It is thicker in boys, in both large and small joints, and in both genders it gradually reduces with age [13,15-17].

US measurement of cartilage thickness in the joints of healthy children appears to have a good inter- and intra-observer agreement for the knee, fairly good or acceptable for the metacarpophalangeal (MCP) joints, ankle and proximal interphalangeal (PIP) joints, but not for the wrist joint [15]. Difficulties seems to be related to the positioning of the joint and the transducer that may affect the measurement, but also challenges in differentiation between the articular cartilage and immature growth cartilage in some complicated anatomical regions [15]. By comparison with the MRI as a gold standard for quantifying the cartilage thickness, a good level of agreement has been found for the knee, ankle, MCP and PIP joints, but again, not for the wrist [16].

Diverse experts have published US measurements of joint cartilage thickness in healthy children at different ages $[13,15-18]$ in order to document age and sexrelated standard reference values. But their findings need to be validated for larger and varied populations, so that a nomogram covering a wide range of age and joints can be constructed.

Physiologicaly, blood vessels may exist at the epiphysis and metaphysis of long bones, in the intra-cartilaginous regions of the small bones and patella, and in the fat pad, and more frequently in 2- to 12-year-old children [2,7]. A power Doppler signal was found in all healthy children and all US scans were performed in a recent study by experienced international ultrasonographers with the highest number of vessels and vessel dots detected in the wrist joint, followed by the tibiotalar and knee joints [7]. For the small peripheral joints, such as MCP II, the detected vessels were the fewest, with only moderate intra- and inter-observer agreement. It was explained by the very low blood flow velocity of the normal vessels in these areas and the possible influence of the environmental temperature and children's activity before the examination [7]. The presence of this physiological vascularization may bean important source of misinterpretation for the sonographers used to adult MSUS examination.
Small amounts of sinovial fluid may also be identified in some normal joints in children.Visible fluid accumulation was demonstrated on MSUS in the knee recesses, at the MCP and PIP synovial recesses and/or the flexor tendon sheaths $[13,19]$. Fluid within the suprapatellar recess of the knee (less than $3 \mathrm{~mm}$ in thickness) was commonly found in almost all age groups and better visualized during contraction of the quadriceps muscles $[13,20]$. None of the healthy children investigated showed joint hip or anterior elbow joint fluid [19-21].

US standards according to age in healthy children have been published so far for the hip joint [22,23] and recently, for the shoulder [21] and wrist [24], which can be beneficial for defining the pathologic changes in every day practice.

The characteristic US appearance of normal tendons, muscles and ligaments is similar with that of adults. But unlike adults, normal tendons may demonstrate visible blood vessels with Dopppler in young children and mild peritendinous vascularity may be seen in children 10-13 years of age [20].

Entheses were also evaluated in healthy volunteers of pediatric ages. Still there are no references for age and gender-related normal appearance of tendon insertions, vascularity expected at the entheses in the growing child [20]. Furthermore it remains normal US enthesis in children to be defined. Jousse-Joulin et al found that tendon thickness at enthesis insertion in children varied significantly with age, but was not influenced by gender [25].Also, the aspect of the bone-cartilage interface varied according to whether the ossification centre had developed within the cartilage: wavy in younger patients, then rounded and, finally, hyperechoic at puberty [25]. Important to say is that enthesalgia may be present in up to $1 / 3$ of healthy school-age children $[20,26]$.

\section{Pathological US findings in patients with different rheumatologic conditions}

The spectrum of pathological conditions for which MSUS has applicability are listed in table I. By far, the most common use of MSUS in pediatric rheumatology is in JIA patients. Furthermore, MSUS isused for the evaluation of other inflammatory and degenerative arthropathies or MSK infections with diagnostic or therapeutic intention. Radiologists investigate developmental dysplasia of the hip or congenital foot deformity in infants, sports injuries, various trauma and infection.

\section{Juvenile Idiopathic Arthritis}

JIA refers to a group of heterogeneous conditions involving chronic joint inflammation of unknown cause, with the onset before the age of 16 . The current Interna- 
Table I. Applications of MSUS in pediatrics [10]

Pediatric rheumatology
Juvenile idiopathic arthritis
Juvenile systemic lupus erythematosus
Transient synovitis of the hip
Traumatisms
Tendon/muscle/articular lesions
Fractures
Baker cysts
Infections
$\quad$ Cellulitis and subcutaneous abscess
$\quad$ Pyomyositis
Osteomyelitis
Septic arthritis
Developmental and congenital disorders
$\quad$ Developmental dysplasia of the hip
$\quad$ Osteochondroses
$\quad$ Tumors
Injuries
Congenital club foot
Foreign bodies
Tumors
Synovial/ mucous cysts
Hemangiomas / lymphangiomas / arteriovenous malformations
Polid tumors

tional League Against Rheumatism (ILAR) classification system comprises seven different types of JIA, based on the number of affected joints and the presence of extraarticular manifestations [27,28].

As in rheumatoid arthritis (RA), MSUS had shown great potential for early diagnosis and extension of arthritis in JIA, as well as monitoring inflammation (synovitis, tenosynovitis and enthesitis) and peripheral joint damage (erosions)[29,30]. Therefore, EULAR and PReS experts recommend the use of MSUS in this clinical setting, in addition to the physical examination [8]. Also, preliminary definitions for the sonographic features of synovitis in children have been recently proposed by the OMERACT Ultrasound Task Forcein order to support MSUS in the routine clinical assessment [3]. Accordingly, synovial effusion was defined as an abnormal, intraarticular, anechoic or hypoechoic material that is displaceable [3]. Synovial hypertrophy was defined as an abnormal, intraarticular, hypoechoic material that is non-displaceable [3]. Color/power Doppler signals must be detected within synovial hypertrophy to be considered as a sign of synovitis [3]. Another important step for the OMERACT Ultrasound Task Force should be the elaboration and validation of US definitions and assessment techniques for tenosynovitis, bursitis, enthesitis, bone and cartilage damage in children. Till then, the OMERACT definitions for these specific pathological features developed for adult patients [31-33] may also assist in the pediatric clinical practice. However sonographers need to be cautious about the presence of physiological vascularization in children. Like in adults, erosions, tenosynovitis and enthesitis need to be verified in two perpendicular scanning planes.

MSUS in the diagnosis of JIA

For many years now, there is supporting evidence that MSUS is more sensitive than physical examination and plain radiography for the detection of synovitis in children with JIA [34-38]. These findings have an implication in classifying the patients into the ILAR subtype of JIA and therefore therapeutic interventions. Furthermore, revealing subclinical synovitis in patients with JIA in clinical remission, may affect the follow up. In such cases, Doppler US should be considered to better differentiate the active subclinical synovitis with destructive potential from the chronic inactive fibrotic synovial tissue. In a previous study, the presence of subclinical synovitis did not predict subsequent synovitis flare [35]. However, more recently, patients with JIA in clinical remission with positive power Doppler signal in joint US have an increased rate of clinical flare [39].

Beside an accurate diagnosis of synovial joint proliferation and joint effusion, MSUS may also effectively assist physicians in the differential diagnosis of patients presenting with swollen ankles or wrists. These are regions with complex anatomy with regard to periarticular structures. For instance, Rooney et al investigated a cohort of 34 children with 49 clinically swollen ankles in order to characterize the distribution of the inflammation process (articular or tenosinovial). They found that tenosynovitis, sometimes in isolation, was the dominant finding ( $71 \%$ of ankles had tenosynovitis and $39 \%$ had tenosynovitis alone) and that only $29 \%$ of swollen ankles had a tibiotalar effusion alone [40]. Their findings may have important implications for local steroid injection therapy in such cases.

Quantification of synovitis on US has not been standardized yet. Different US scoring systems have been used in JIA studies so far for scoring synovial hypertrophy or the degree of pannus vascularity. They were mainly based on the semiquantitative scales previously proposed by the OMERACT in RA [41]. Collado et al have proposed a new scoring system of paediatric synovitis (PedSynS) for the elbow, radiocarpal, tibiotalar, mid-foot and finger joints [42]. The PedSynS consists of a 4-point semiquantitative scale of grey scale synovitis: 0 , absence, normal joint recess; 1 , mild, synovitis filling the joint recess between periarticular epiphyses that leads to change 
from the angle-shaped recess to a plateau-shaped recess; 2 , moderate, convex shape of the joint recess without extension over thebone diaphysis; and 3, marked, convex shape of the joint recess with extension to at least one of the bone diaphyses [42]. The authors also demonstrated that a reduced 10-joints PDUS assessment (bilateral knee, ankle, wrist, elbow and the $2 \mathrm{MCP}$ joints) is valid and feasible for the assessment of synovitis in JIA for clinical practice [42]. But, general consensus is required for this subject.

Enthesitis is a rare phenomenon in JIA that characterizes juvenile enthesitis-related arthritis (ERA). There is currently no gold standard imaging technique to detect enthesitis [43]. Detection of peripheral enthesitis in children with ERA can be improved with US $[44,45]$. In their study, Weiss at al showed that, in comparison to US, physical examination with dolorimeter is a poor diagnostic test for enthesitis [45]. But, US diagnosis of enthesitis in children with JIA will probably improve when a standardized, reliable and reproducible definition for normal enthesis and also for enthesitis will be available.

MSUS in monitoring the disease activity and

response to treatment

MSUS imaging can also play a key role in guiding steroid injections into joints, tendon sheathsand even tendon insertions (entheses) [46-48]. Local corticosteroid (preferably triamcinolone hexacetonide) injections are a common treatment for young patients with JIA, well tolerated and effective for several months, used particularly in the oligoarthritis subset of JIA. They can also be used when systemic anti-inflammatory treatment fails to control isolated arthritis or tenosynovitis. The benefits and safety of MSUS with PD as guidance of steroid injections has been demonstrated in the symptomatic wrist and ankle regions of JIA patients by Laurell et al $[46,47]$. Using US guidance allows full needle visualization in real-time, including the needle tip. This technique offers greater clinical efficacy by ensuring the correct injection of the therapeutic substance and thus reducing the risk of complications. In clinical practice, the most commonly injected tendon sheaths seem to be the tibialis posterior, peroneus longus and peroneus brevis, as reported in a recent observational study [48]. No major complications were observed [48].

Having the ability of directly visualizing the synovial joint proliferation and joint effusion, MSUS has the potential for monitoring the response to therapy. But incorporating US in the JIA core set of outcome measures of clinical trials is under evaluation. Also, implementing ultrasound in remission assessment is an interesting area of discussion, since the traditional criteria only include clinical and laboratory parameters.
High-resolution US can also be used for monitoring structural joint damage such as bone erosions and cartilage loss in accessible surfaces. Research examining the validity of US in the assessment of bone erosions mostly comes from RA adults. In JIA, there may be some challenges in interpreting bone surface irregularities found on MSUS as pathological or not. For instance, in the wrists of healthy children, there may be bony depressions that resemble erosions, as demonstrated by MRI studies $[49,50]$. Likewise, evaluating cartilage loss in JIA patients may be difficult, since cartilage thickness normally decreases with skeletal maturity [29]. Even so, MSUS is a reliant tool for visualization of childhood femoral cartilage in JIA, highly correlated to MRI measurements [51]. For instance, the authors recommend the intercondylar notch of the distal femoral cartilage to be measured when evaluating the cartilage thickness of the knee [51].

In the meantime, according to EULAR-PReS consensus-based recommendations, MSUS and MRI can be used to detect damage in JIA at an earlier time point than conventional radiography [8], the current reference for detection of structural damage in JIA [29].

\section{Juvenile Systemic Lupus Erythematosus}

Pediatric patients with systemic lupus erythematosus (SLE) may also experience musculoskeletal involvement: arthritis, arthralgia, myalgia, myositis, tenosynovitis, bone fragility fractures or osteonecrosis, secondary pain amplification [52]. Unlike JIA, arthritis in juvenile SLE is typically non-erosive and non-deforming [52]. Rarely, Rhupus syndrome and Jaccoud's arthropathy may be present in children [53]. Therefore, MSUS can be used in juvenile SLE as a first method for evaluation of synovitis, tenosynovitis and even bone erosions. Recent US studies report bone erosions in SLE adult patients and not only in the rhupus subtype $[54,55]$.

\section{Transient synovitis of the hip}

Transient synovitis (TS) of the hip refers to a selflimiting acute inflammatory condition, in fact the most common cause of acute hip pain and limp in children aged 3-8 years [56,57]. The etiology of TS is uncertain and therefore the diagnosis is made by the exclusion of different infectious, orthopedic, neoplastic or other inflammatory disorders [57]. When plain radiographs of this area are normal, MSUS is often requested to confirm the presence of the hip joint effusion. An effusion is validated if US demonstrates capsular distension greater than $2 \mathrm{~mm}$. The joint space widening is measured at the point of maximum enlargement, between the iliopsoas muscle and the anterior surface of the femoral neck, perpendicular to the bone surface $[57,58]$. TS can have an identical US aspect with septic arthritis (SA). A definitive diagnosis can only be made by US-guided joint aspiration and 
synovial fluid culture. Kocher criteria (history of fever, non-weight-bearing, elevated inflammatory markers and peripheral leukocytosis [59]) can help in differentiating those patients with a high suspicion of SA that requires arthrocentesis. TS has a benign clinical course that is resolved with supportive treatment, but a second US evaluation is recommended after 10-15 days in order to ascertain the disappearance of the hip effusion [57].

\section{Septic arthritis}

Pediatric septic arthritis is a challenging clinical issue where MSUS has an important role in the diagnosis workup. Any joint can be affected but the hip and knee joints are the most common [60]. US can visualize joint effusions in suspected septic arthritis. Some US findings may be relevant for SA, such as intraarticular echogenic debris or presence of hyperechogenic spots, but cannot safely distinguish between septic and non-septic arthritis [61]. Yet, it can guide the arthrocentesis for synovial fluid aspiration.

\section{Conclusions}

Over the last years, MSUS has accumulated a significant positive impact in diagnostic accuracy and monitoring children with rheumatologic disorders. More evidence about normal age-related US appearance ofmusculoskeletal tissues and structures in children is required. Since US examination is relatively operator dependent, adhering to proper US techniques, performing standardized scanning procedures and using specific US definitions for pediatric populationare mandatory in order to limit false interpretations. Using educational programs in pediatric musculoskeletal US, along with independent bedside practice, mentor guidance and access to specific pathology can really help clinicians to develop essential knowledge and skills to integrate ultrasound into routine clinical practice.

\section{References}

1. Möller I, Janta I, Backhaus M, et al. The 2017 EULAR standardised procedures for ultrasound imaging in rheumatology. Ann Rheum Dis 2017;76:1974-1979.

2. Collado P, Vojinovic J, Nieto JC, et al; Omeract Ultrasound Pediatric Group. Toward standardized musculoskeletal ultrasound in pediatric rheumatology: normal agerelated ultrasound findings. Arthritis Care Res (Hoboken) 2016;68:348-356.

3. Roth J, Ravagnani V, Backhaus M, et al; OMERACT Ultrasound Group. Preliminary Definitions for the Sonographic Features of Synovitis in Children. Arthritis Care Res (Hoboken) 2017;69:1217-1223 .
4. Roth J, Jousse-Joulin S, Magni-Manzoni S, et al; Outcome Measures in Rheumatology Ultrasound Group. Definitions for the sonographic features of joints in healthy children. Arthritis Care Res (Hoboken) 2015;67:136-142.

5. Collado P, Jousse-Joulin S, Alcalde M, Naredo E, D'Agostino MA. Is ultrasound a validated imaging tool for the diagnosis and management of synovitis in juvenile idiopathic arthritis? A systematic literature review. Arthritis Care Res (Hoboken) 2012;64:1011-1019.

6. Lanni S, Wood M, Ravelli A, Magni Manzoni S, Emery $\mathrm{P}$, Wakefield RJ. Towards a role of ultrasound in children with juvenile idiopathic arthritis. Rheumatology (Oxford) 2013;52:413-420.

7. Windschall D, Collado P, Vojinovic J, et al; OMERACT paediatric ultrasound subtask force. Age-related vascularization and ossification of joints in children: an international pilot study to test multi-observer ultrasound reliability. Arthritis Care Res (Hoboken) 2017. doi:10.1002/ acr.23335.

8. Colebatch-Bourn AN, Edwards CJ, Collado P, et al. EULAR-PReS points to consider for the use of imaging in the diagnosis and management of juvenile idiopathic arthritis in clinical practice. Ann Rheum Dis 2015;74:1946-1957.

9. Wakefield RJ, D’Agostino MA. Use of ultrasonography in the pediatric patient. In: Wakefield RJ, D'Agostino MA (eds.). Essential Applications of Musculoskeletal Ultrasound in Rheumatology. 1st ed. Saunders Elsevier, Philadelphia 2010:207-210.

10. Fodor D. Ecografia musculoscheletala în pediatrie. In: Fodor D. Aplicații clinice ale ecografiei musculoscheletale. Ed. Medicală, București 2018:181-224.

11. Ortega N, Behonick DJ, Werb Z. Matrix remodeling during endochondral ossification. Trends Cell Biol 2004;14:86-93.

12. Kan JH, Strouse PJ. Embryology, anatomy, and normal findings. Available from: https://clinicalgate.com/embryology [Accessed 5th January 2018].

13. Windschall D, Trauzeddel R, Haller M, et al; Imaging Working Group of the German Society of Rheumatology in Childhood and Adolescense (GKJR). Pediatric musculoskeletal ultrasound: age- and sex-related normal B-mode findings of the knee. Rheumatol Int 2016;36:1569-1577.

14. Windschall D, Pommerenke M, Haase R. Ultrasound assessment of the skeletal development of the proximal tibial, proximal femoral, and distal femoral epiphyses in premature and mature newborns. Ultrasound Med Biol 2016;42:451-458.

15. Spannow AH, Pfeiffer-Jensen M, Andersen NT, Stenbog $\mathrm{E}$, Herlin T. Inter -and intraobserver variation of ultrasonographic cartilage thickness assessments in small and large joints in healthy children. Pediatr Rheumatol Online J 2009; 7:12.

16. Spannow AH, Stenboeg E, Pfeiffer-Jensen M, et al. Ultrasound and MRI measurements of joint cartilage in healthy children: a validation study. Ultraschall Med 2011;32 Suppl $1:$ S110-S116.

17. Spannow AH, Stenboeg E, Pfeiffer-Jensen M, Herlin T. Ultrasound measurement of joint cartilage thickness in large 
and small joints in healthy children: a clinical pilot study assessing observer variability. Pediatr Rheumatol Online J 2007;5:3.

18. Spannow AH, Pfeiffer-Jensen M, Andersen NT, Herlin T, Stenbøg E. Ultrasonographic measurements of joint cartilage thickness in healthy children: age- and sex-related standard reference values. J Rheumatol 2010;37:25952601.

19. Collado P, Naredo E, Calvo C, Crespo M. Assessment of the joint recesses and tendon sheaths in healthy children by high resolution B-mode and power Doppler sonography. Clin Exp Rheumatol 2007;25:915-921.

20. Chauvin NA, Ho-Fung V, Jaramillo D, Edgar JC, Weiss PF. Utrasound of the joints and entheses in healthy children. Pediatr Radiol 2015;45:1344-1354.

21. Trauzeddel R, Windschall D, Trauzeddel RF, et al. Arthrosonographic reference values of the shoulder joint in healthy children and adolescents: a cross-sectional multicentre ultrasound study. Klin Padiatr 2017;229:293-301.

22. Robben SG, Lequin MH, Diepstraten AF, den Hollander JC, Entius CA, Meradji M. Anterior joint capsule of the normal hip and in children with transient synovitis: US study with anatomic and histologic correlation. Radiology 1999;210:499-507.

23. Rohrschneider WK, Fuchs G, Tröger J. Ultrasonographic evaluation of the anterior recess in the normal hip: a prospective study on 166 asymptomatic children. Pediatr Radiol 1996;26:629-634.

24. Rosendahl K, Bruserud IS, Oehme N, et al. Normative ultrasound references for the paediatric wrist; dorsal soft tissues. RMD Open 2018;4:e000642.

25. Jousse-Joulin S, Cangemi C, Gerard S, et al. Normal sonoanatomy of the paediatric entheses including echostructure and vascularisation changes during growth. Eur Radiol 2015;25:2143-2152.

26. Sherry DD, Sapp LR. Enthesalgia in childhood: site-specific tenderness in healthy subjects and in patients with seronegative enthesopathic arthropathy. J Rheumatol 2003;30:1335-1340.

27. Petty RE, Southwood TR, Manners P, et al; International League of Associations for Rheumatology. International League of Associations for Rheumatology classification of juvenile idiopathic arthritis: second revision, Edmonton, 2001. J Rheumatol 2004;31:390-392.

28. Ravelli A, Martini A. Juvenile idiopathic arthritis. Lancet 2007;369:767-778.

29. Collado P, Malattia C. Imaging in paediatric rheumatology: Is it time for imaging? Best Pract Res Clin Rheumatol 2016;30:720-735.

30. Azouz EM. Arthritis in children: conventional and advanced imaging. Semin Musculoskelet Radiol 2003;7:95102.

31. Terslev L, Naredo E, Iagnocco A, et al. Outcome Measures in Rheumatology Ultrasound Task Force. Defining enthesitis in spondyloarthritis by ultrasound: results of a Delphi process and of a reliability reading exercise. Arthritis Care Res (Hoboken) 2014;66:741-748.
32. Wakefield RJ, Balint PV, Szkudlarek M, et al; OMERACT 7 Special Interest Group. Musculoskeletal ultrasound including definitions for ultrasonographic pathology. J Rheumatol 2005;32:2485-2487.

33. Bruyn GA, Hanova P, Iagnocco A, et al; OMERACT Ultrasound Task Force. Ultrasound definition of tendon damage in patients with rheumatoid arthritis. Results of a OMERACT consensus-based ultrasound score focussing on the diagnostic reliability. Ann Rheum Dis 2014;73:1929-1934.

34. Magni-Manzoni S. Ultrasound in juvenile idiopathic arthritis. Pediatr Rheumatol Online J 2016;14:33.

35. Magni-Manzoni S, Sciré CA, Ravelli A, et al. Ultrasounddetected synovial abnormalities are frequent in clinically inactive juvenile idiopathic arthritis, but do not predict a flare of synovitis. Ann Rheum Dis 2013;72:223-228.

36. Magni-Manzoni S, Epis O, Ravelli A, et al. Comparison of clinical versus ultrasound-determined synovitis in juvenile idiopathic arthritis. Arthritis Rheum 2009;61:1497-1504.

37. Damasio MB, Malattia C, Martini A, Toma P. Synovial and inflammatory diseases in childhood: role of new imaging modalities in the assessment of patients with juvenile idiopathic arthritis. Pediatr Radiol 2010;40:985-998.

38. Sparchez M, Fodor D, Miu N. The role of power Doppler ultrasonography in comparison with biological markers in the evaluation of disease activity in juvenile idiopathic arthritis. Med Ultrason 2010;12:97-103.

39. Miotto E Silva VB, Mitraud SAV, Furtado RNV, Natour J, Len CA, Terreri MTSELRA. Patients with juvenile idiopathic arthritis in clinical remission with positive power Doppler signal in joint ultrasonography have an increased rate of clinical flare: a prospective study. Pediatr Rheumatol Online J 2017;15:80.

40. Rooney ME, McAllister C, Burns JF. Ankle disease in juvenile idiopathic arthritis: ultrasound findings in clinically swollen ankles. J Rheumatol 2009;36:1725-1729.

41. Naredo E, Bonilla G, Gamero F, Uson J, Carmona L, Laffon A. Assessment of inflammatory activity in rheumatoid arthritis: a comparative study of clinical evolution with grey scale and power Doppler ultrasonography. Ann Rheum Dis 2005;64:375-381.

42. Collado P, Naredo E, Calvo C, et al; ECO-JIA Study Group. Reduced joint assessment vs comprehensive assessment for ultrasound detection of synovitis in juvenile idiopathic arthritis. Rheumatology (Oxford) 2013;52:1477-1484.

43. Micu CM, Fodor D. Concepts in monitoring enthesis in patients with spondylarthritis- the role of musculoskeletal ultrasound. Med Ultrason 2016;18:82-89.

44. Jousse-Joulin S, Breton S, Cangemi C, et al. Ultrasonography for detecting enthesitis in juvenile idiopathic arthritis. Arthritis Care Res (Hoboken) 2011;63:849-855.

45. Weiss PF, Chauvin NA, Klink AJ, et al. Detection of enthesitis in children with enthesitis-related arthritis: dolorimetry compared to ultrasonography. Arthritis Rheumatol 2014;66:218-227.

46. Laurell L, Court-Payen M, Nielsen S, Zak M, Boesen M, Fasth A. Ultrasonography and color Doppler in juvenile idiopathic arthritis: diagnosis and follow-up of ultrasound- 
guided steroid injection in the ankle region. A descriptive interventional study. Pediatr Rheumatol Online J 2011;9:4.

47. Laurell L, Court-Payen M, Nielsen S, Zak M, Fasth A. Ultrasonography and color Doppler in juvenile idiopathic arthritis: diagnosis and follow-up of ultrasound-guided steroid injection in the wrist region. A descriptive interventional study.Pediatr Rheumatol Online J 2012;10:11.

48. Peters S, Laxer RM, Connolly BL, Parra DA. Ultrasoundguided steroid tendon sheath injections in juvenile idiopathic arthritis: a 10-year single-center retrospective study. Pediatric Rheumatology 2017;15:22.

49. Avenarius DM, Ording Müller LS, Eldevik P, Owens CM, Rosendahl K. The paediatric wrist revisited--findings of bony depressions in healthy children on radiographs compared to MRI. Pediatr Radiol 2012;42:791-798.

50. Avenarius DF, Ording Müller LS, Rosendahl K. Erosion or normal variant? 4-year MRI follow-up of the wrists in healthy children. Pediatr Radiol 2016;46:322-330.

51. Pradsgaard DO, Fiirgaard B, Spannow AH, Heuck C, Herlin T. Cartilage thickness of the knee joint in juvenile idiopathic arthritis: comparative assessment by ultrasonography and magnetic resonance imaging. J Rheumatol 2015;42:534-540.

52. Levy D, Kamphuis S. Systemic Lupus Erythematosus in Children and Adolescents. Pediatr Clin North Am 2012;59:345-364.

53. Cavalcante EG, Aikawa NE, Lozano RG, Lotito AP, Jesus AA, Silva CA. Chronic polyarthritis as the first manifesta- tion of juvenile systemic lupus erythematosus patients. Lupus 2011;20:960-964.

54. Piga M, Saba L, Gabba A, et al. Ultrasonographic assessment of bone erosions in the different subtypes of systemic lupus erythematosus arthritis: comparison with computed tomography. Arthritis Res Ther 2016;18:222.

55. Zayat AS, Md Yusof MY, Wakefield RJ, Conaghan PG, Emery P, Vital EM. The role of ultrasound in assessing musculoskeletal symptoms of systemic lupus erythematosus: a systematic literature review. Rheumatology (Oxford) 2016;55:485-494.

56. Do TT. Transient synovitis as a cause of painful limps in children. Curr Opin Pediatr 2000;12:48-45.

57. Pauroso S, Di Martino A, Tarantino CC, Capone F. Transient synovitis of the hip: Ultrasound appearance. Minipictorial essay. J Ultrasound 2011;14:92-94.

58. DiPietro MA, Leschied JR. Pediatric musculoskeletal ultrasound. Pediatr Radiol 2017;47:1144-1154.

59. Kocher MS, Mandiga R, Zurakowski D, Barnewolt C, Kasser JR. Validation of a clinical prediction rule for the differentiation between septic arthritis and transient synovitis of the hip in children. J Bone Joint Surg Am 2004;86:1629-1635.

60. Ahmed H, Dix-Peek S, Martin N, Hoffman EB. Septic arthritis in children: A 20-year study. J Bone Joint Surg Br 2005;87-B:279.

61. Zamzam MM. The role of ultrasound in differentiating septic arthritis from transient synovitis of the hip in children. $J$ Pediatr Orthop B 2006;15:418-422. 